\title{
木本植物木质部栓塞修复机制: 研究进展与问题
}

\author{
金 鹰 王传宽 ${ }^{*}$ 周正虎
}

东北林业大学生态研究中心, 哈尔滨 150040

摘 要 维持木本植物体内长距离的水分运输对于植物生存、生长和发育非常重要, 但因水分在木质部张力状态下处于亚稳 定状态而易发生空穴化和栓塞, 导致水力导度降低、生产力下降、甚至植物死亡。面对水分胁迫诱导的空穴化, 植物可通过 形成抵抗空穴化的解剖结构降低栓塞发生频率, 或(和)通过活跃的代谢修复栓塞, 其中对木质部栓塞及其修复的发生频率、 条件、机制等的认识仍有很大分歧。为此, 该文首先综述了木质部栓塞修复过程及时间动态、木质部栓塞形成及修复的发生 频率。然后, 总结了木质部导管“新的再充水”栓塞修复过程中的4种主要假说: (1)渗透调节假说; (2)反渗透调节假说; (3)韧皮 部驱动再充水假说; (4)韧皮部卸载假说。在此基础上, 比较了针叶树种和木本被子植物木质部栓塞形成与修复的差异, 并分 析了木质部栓塞阻力与修复能力之间的权衡关系。最后, 提出了木本植物木质部栓塞与修复研究的 4 个优先研究问题: (1)改进 木质部栓塞测定技术; (2)验证“新的再充水”栓塞修复机制假说及引发木质部再充水的信号; (3)阐明木质部栓塞与修复特性的 树种间差异及其可能的权衡关系; (4)加强碳代谢和水通道蛋白表达与木质部栓塞及其修复关系的生理生化研究。

关键词木质部栓塞; 栓塞修复; 栓塞频率; 新的再充水; 㓞皮部卸载

引用格式: 金鹰, 王传宽, 周正虎 (2016). 木本植物木质部栓塞修复机制: 研究进展与问题. 植物生态学报, 40, 834-846. doi: 10.17521/cjpe.2016. 0118

\section{Mechanisms of xylem embolism repair in woody plants: Research progress and questions}

JIN Ying, WANG Chuan-Kuan*, and ZHOU Zheng-Hu

Center for Ecological Research, Northeast Forestry University, Harbin 150040, China

\section{Abstract}

To maintain long-distance water transport in woody plants is critical for their survival, growth and development. Water under tension is in a metastable state and prone to cavitation and embolism, which leads to loss of hydraulic conductance, reduced productivity, and eventually plant death. In face to water stress-induced cavitation, plants either reduce frequency of embolism occurrence through cavitation resistance with specialized anatomical structure, or/and form a metabolically active embolism repair mechanism. For the xylem embolism and repair, however, there are controversies regarding the occurring frequency, conditions and underlying mechanisms. In this review paper, we first examined the process, temporal dynamics and frequency of xylem embolism and repair. Then, we summarized hypotheses for the mechanisms of the novel refilling in xylem embolism repair, including the osmotic hypothesis, the reverse osmotic hypothesis, the phloem-driven refilling hypothesis, and the phloem unloading hypothesis. We further compared differences in xylem embolism and repair between conifers and angiosperms tree species, and examined the trade-offs between cavitation resistance and xylem recovery performance. Finally, we proposed four priorities in future research in this field: (1) to improve measuring technology of xylem embolism; (2) to test hypotheses for the mechanisms of the novel refilling in xylem embolism repair and the signal triggering xylem refilling; (3) to explore species-specific trait differences related to xylem embolism and repair and their underlying trade-off relationships; and (4) to enhance studies on the relationship between the involvement of carbon metabolism and aquaporins expression in xylem embolism and repair.

Key words xylem embolism; embolism repair; embolism frequency; novel refilling; phloem unloading

Citation: Jin Y, Wang CK, Zhou ZH (2016). Mechanisms of xylem embolism repair in woody plants: Research progress and questions. Chinese Journal of Plant Ecology, 40, 834-846. doi: 10.17521/cjpe.2016.0118

收稿日期Received: 2016-03-30 接受日期Accepted: 2016-07-19

* 通信作者Author for correspondence (E-mail: wangck@nefu.edu.cn) 
高等木本植物具有发达的水分运输系统, 能把 土壤中的水分高效地运输到叶片, 从而保证叶片正 常的水分供应(Meinzer \& McCulloh, 2013)。因此, 维持根-茎-叶木质部内水分运输的连续性对于植物 的生存、生长和发育非常重要(Ogasa et al., 2013)。 根据内聚力-张力理论, 植物体内水分在负压下以 水柱的形式在木质部导管中向上运输, 张力状态下 的水分处于亚稳态; 当木质部负压超过一定阈值 时, 水柱即会断裂, 气泡便会从气体填充的导管吸 出, 通过导管间的纹孔膜进入原本充水的管道, 并 逐渐扩张, 阻碍导管的水分运输, 最终形成栓塞 (Sperry et al., 1988; Jansen et al., 2009; 李荣等, 2015)。这种空穴化诱导的栓塞会引起木质部水力导 度降低，进而导致叶片水势下降和气孔关闭，限制 植物的光合作用和生产力, 最终导致灾难性的水力 失调(Zwieniecki \& Holbrook, 1998; Salleo et al., 2000)。

长期以来, 人们认为木质部空穴化和栓塞是极 少发生且不可逆的过程; 植物通过产生新的木质部 导管, 恢复根-茎-叶水力连续体的完整性, 保证充 足的水力能力以维持稳定的光合作用(Brodribb et $a l ., 2010)$ 。近年来, 越来越多的研究认为, 空穴化和 栓塞是植物体内极易发生的常见事件, 且在一定程 度上这种频繁发生的栓塞在木质部负压下也能被及 时修复(Secchi \& Zwieniecki, 2010, 2011; Nardini et al., 2011; Johnson et al., 2012)。然而, 由于研究方 法、物种、时间分辨率等的不同, 对木质部频繁发 生栓塞并修复的观点仍有很大争议 (Brodersen \& McElrone, 2013; McCulloh \& Meinzer, 2015)。阐明木 本植物木质部栓塞的发生特性及修复机制, 有利于 评价不同功能型植物对干旱胁迫的应对, 对预测气 候变化情景下植物的分布、篮选抗旱植物等均有重 要意义。为此, 本文首先综述了木质部栓塞修复过 程及时间动态、木质部栓塞及修复的发生频率。然 后, 总结了木质部栓塞修复机制一一新的再充水 (novel refilling), 主要包括4种假说: (1)渗透调节假 说; (2)反渗透调节假说; (3)韧皮部驱动再充水假说; (4)韧皮部卸载假说。在此基础上, 比较了针叶树种 和木本被子植物木质部栓塞及修复的差异, 探索了 木质部栓塞阻力与修复能力之间的权衡关系。最后, 提出了木本植物木质部栓塞与修复研究的 4 个优先 研究问题。

\section{1 木质部栓塞修复过程及时间动态}

早期研究认为, 植物木质部栓塞修复只有在木 质部水势为正压或者稍负时才能发生(Zimmermann, 1983)。然而, 随着对栓塞修复过程研究的深 入, 更多的证据表明在张力状态下木质部栓塞也可 修复(Zwieniecki \& Holbrook, 2009; Brodersen et al., 2010)。张力状态下的栓塞修复过程主要包括3个步 骤: (1)水分移动到再充水导管; (2)再充水导管与功 能性导管的水力隔离, 以确保产生消解或驱逐气体 所需要的正压力; (3)再充水过程完成后, 水力隔离 的解除(Holbrook \& Zwieniecki, 1999)。

正常情况下，导管周围的薄壁细胞向木质部持 续释放少量可溶性碳水化合物(Zwieniecki \& Holbrook, 2009)。在功能性导管中, 这些溶质会被蒸腾 液流带走, 从而使薄壁细胞壁上的糖浓度保持在低 水平状态。但如果导管发生了空穴化, 糖的积累导 致相关质外体溶质浓度增加, 从而产生栓塞信号。 栓塞导管中溶质的积累使水分通过渗透作用从木质 部薄壁细胞移动到再充水导管, 在导管内壁上形成 水滴(Zwieniecki \& Holbrook, 2000)。在这个过程中, 纹孔起到了使再充水导管与周围功能性导管水力隔 离的作用, 从而阻止这些水滴被功能型导管吸走 (Zwieniecki \& Holbrook, 2000; Vesala et al., 2003)。 随着水滴聚结并逐渐扩大, 再充水导管内部的压力 增加, 驱使气体消解于溶液中或通过小孔隙穿越导 管壁而被赶到细胞间隙中去。当再充水导管壁上的 溶质浓度降至栓塞前的水平时 (由于蒸腾液流的恢 复, 稀释作用提供了再充水过程的关闭信号), 再充 水过程结束。在栓塞导管完成再充水后, 水分进入 具缘纹孔通道。向外打开的具缘纹孔腔起到阀门作 用, 一旦导管腔内压力超过进入具缘纹孔腔的压力 阈值, 则功能性导管与再充水导管会再次相连, 解 除水力隔离(Zwieniecki \& Holbrook, 2009)。

Zwieniecki和Holbrook (2009)的研究指出: 无 论是自然形成的, 还是实验诱导的栓塞, 均可在短 时间内恢复(一般不到 1 h; Salleo et al., 2004; Hölttä et al., 2006)。此外, 栓塞修复的时间长短可能与植 物遭受的水分胁迫程度有关 (Zwieniecki et al., 2013)。Secchi和Zwieniecki (2011)对Populus trichocarpa 的研究发现: 在适度水分胁迫下可快速修复 栓塞 $(<2 \mathrm{~h})$, 但在极端水分胁迫下栓塞修复时间较 长 $(>20 \mathrm{~h})$ 。也有研究认为: 植物快速修复栓塞的实 
验证据均是来自于破坏性的取样方法; 而使用核磁 共振成像技术(magnetic resonance imaging, MRI)和 $\mathrm{X}$ 射线显微层析技术(X-ray microtomography, micro-CT)的活体观测方法则发现导管的栓塞修复速 率较慢(Holbrook et al., 2001; Scheenen et al., 2007; Brodersen et al., 2010)。因此, 未来研究应着重开展 基于无损可视化技术的研究, 以阐明活体状态下植 物栓塞形成和修复的时间动态。

\section{2 木质部栓塞和修复的发生频率}

在干旱胁迫情况下, 栓塞的积累将最终导致木 质部水分运输失调, 甚至使植物死亡(Brodribb \& Cochard, 2009)。木本植物尤其是高大乔木面对木质 部栓塞对其生存构成的威胁如何应对：是通过高空 穴化阻力避免栓塞发生(Salleo et al., 2000), 还是通 过高修复能力修复频繁发生的栓塞(Salleo et al., 1996, 2004; Tyree et al., 1999; Bucci et al., 2003; Hacke \& Sperry, 2003)? 目前的研究主要形成以下 两种推论: (1)一种认为许多木本植物的栓塞和修复 是常规发生的 (Brodersen \& McElrone，2013; McCulloh \& Meinzer, 2015); (2)另一种认为栓塞只 有在极端水分胁迫条件下才能发生, 且只有在水分 条件好、植物体内存在正压的情况下才能被修复 (Holbrook \& Zwieniecki, 1999; Cochard \& Delzon, 2013; Wheeler et al., 2013)。

木质部对空穴化的脆弱性通常采用脆弱性曲线 确定(Sperry et al., 1988)。早期人们发现植物木质部 脆弱性曲线呈“S”形模式, 即“高-空穴化-阻力”模式 (Cochard \& Delzon, 2013)。这种模式认为: 栓塞是极 少发生的事件, 只有在极端水分胁迫条件下才能发 生(Choat et al., 2012), 而且该栓塞过程是不可逆的, 即一旦发生栓塞就不可修复, 最终将不可避免地导 致植物因水力失调而死亡(Sperry et al., 1988; Holbrook \& Zwieniecki, 1999)。因此, 早期研究者认为: 树木对干旱的容忍能力取决于其抗空穴化能力的高 低(Brodribb \& Cochard, 2009; Brodribb et al., 2010; Urli et al., 2013)。20世纪90年代中期之后越来越多 的研究发现: 很多树种的脆弱性曲线呈 “指数型” 模 式, 即“低-空穴化-阻力”模式(Tyree et al., 1999)。该 模式认为: 树木经常发生栓塞, 甚至在水分状况良 好的条件下也会发生, 而且这种频繁经历的栓塞在 木质部水势为负值时也能快速恢复(Salleo et al.,
1996; Zwieniecki \& Holbrook, 1998; Tyree et al., 1999; Bucci et al., 2003; Hacke \& Sperry, 2003)。因 此, 这个时期普遍认为树木对干旱的容忍能力取决 于其栓塞修复能力的高低。

然而，近年来关于植物有规律的经历栓塞和修 复的过程引起了很大争议(Cochard \& Delzon, 2013; Wheeler et al., 2013; McCulloh \& Meinzer, 2015; Torres-Ruiz et al., 2015; Venturas et al., 2015)。 Wheeler等(2013)认为: 以前支持植物有规律地经历 栓塞和修复的绝大部分实验性证据均是由于实验方 法问题而人为造成的。人们在取样时在水下切割样 本, 以为这样做能保证在切割的瞬间保持木质部管 道系统处于自然张力下, 能准确反映树木原位空穴 化水平。然而, Wheeler等(2013)的研究发现: 这种在 自然张力(负压)下切断枝条的取样方法会人为诱发 空穴化, 增加样本水力导度的损失(tension-cutting artefact), 从而低估诱导树木空穴化所需要的胁迫 水平(McElrone et al., 2012; Cochard et al., 2013; Cochard \& Delzon, 2013)。为此, 在样本离体之前应 该先把样本的张力释放掉(Wheeler et al., 2013)。总 之, Wheeler等(2013)认为, 木质部栓塞和修复并不 是经常发生的, 树木应对空穴化的阻力远比人们想 象的要强, 树木有能力构建一个很好的抵抗空穴化 的管道系统(Cochard \& Delzon, 2013)。

最近, Venturas等(2015)比较了在自然张力下切 割样本和释放张力后切割样本对水力导度损失影响 的差异, 结果表明两种方法无显著差异, 且在自然 张力下切割样本并没有诱导木质部空穴化。他们指 出, Wheeler等(2013)所提的释放张力后切割样本的 方法可能会使导管再充水而恢复栓塞, 产生了再充 水的人为影响(refilling artefact)。Trifilò等(2014)对油 橄榄(Olea europaea)和月桂(Laurus nobilis)采用这 两种方法的比较研究表明, 释放张力切割样本的方 法确实产生了导管再充水的人为影响。且Scoffoni 和Sack (2014)对4个树种叶片水力的研究结果并没 有支持张力诱导栓塞的人为影响。此外, 基于非破 坏性的活体观测方法(如MRI和micro-CT技术)均证 明了栓塞形成和修复循环过程的存在(Holbrook et al., 2001; Brodersen et al., 2010; Zwieniecki et al., 2013)。Brodersen等(2010)利用高分辨率的X射线计 算机断层成像技术观察活体葡萄(Vitis vinifera)植物 茎的水分含量发现, 栓塞导管壁上不仅存在水滴, 
而且水滴大小在再充水过程中还存在动态变化, 支 持了再充水过程的存在。Zwieniecki等(2013)的活体 MRI观测技术研究表明: 适度干旱胁迫诱导的栓塞 可快速地修复(约30 min), 支持了在自然状况下栓 塞和修复循环过程的存在; 但活体状态下这种循环 过程的发生频率尚不清楚。目前, 利用 MRI和 micro-CT技术观测栓塞循环过程的研究均证明了栓 塞后再充水过程的存在, 但关于活体观测栓塞和修 复循环过程频率的证据仍然缺乏。未来的研究需要 关注活体植物自然产生的干旱胁迫导致的栓塞及其 修复循环过程的发生频率。综上所述, 越来越多的 证据支持植物是有规律地经历栓塞和修复, 但并不 是所有物种均能修复栓塞, 这可能与物种解剖结构 有关(Brodersen \& McElrone, 2013; Ogasa et al., 2013; Urli et al., 2013; McCulloh \& Meinzer, 2015; Trifilò et al., 2015)。因此, 探索植物栓塞修复的生理 机制与解剖结构的关系, 对深入理解不同物种和功 能群之间抗旱能力差异很必要。

值得一提的是, 尽管栓塞形成与修复的循环过 程在植物体内可能是常见事件, 但Hacke等(2001)的 研究表明这种反复循环过程可能会导致导管空穴化 疲劳现象, 从而削弱木质部的空穴化阻力; 而这种 空穴化疲劳现象因树种而异, 有些树种较敏感, 而 有些树种有强的容忍性(Hacke et al. 2001)。前者可 能是由于导管间纹孔膜纤维网的损坏或变松, 导致 气体渗透性的增加; 如果是纹孔膜纤维网被损坏, 那么这种削弱影响可能是永久性的; 如果只是由于 微纤丝被拉伸导致纹孔膜纤维网变松, 那么活体植 物很可能恢复拉伸, 并消除这种削弱影响(Plavcová et al., 2012)。然而, Hacke等(2001)的研究全部来自 于离体实验, 而活体植物的栓塞修复的再充水过程 是否能够恢复其最初的空穴化阻力尚未可知。Stiller 和Sperry (2002)研究向日葵(Helianthus annuus)的空 穴化疲劳及其恢复过程发现: 在自然条件下, 活体 植物的空穴化疲劳是可恢复的(Sperry \& Hacke, 2004)。此外, Brodersen等(2013)的研究支持了空穴 化疲劳的观点, 认为经历过栓塞的导管比新的木质 部导管对之后的栓塞更为脆弱, 但栓塞的形成首先 发生在茎髓心周围的导管中, 这可能是源于最老导 管组织(更接近髓心的导管)的纹孔膜遭受反复胁迫 的积累所致, 最终导致其变成非功能性导管(Choat et al. 2005)。

\section{3 木质部栓塞修复机制}

木质部栓塞及修复是植物应对张力状态下水分 运输的内在调节机制。尽管导管栓塞形成是非生物 学过程, 与木质部水柱中张力的大小和木材理化性 质有关, 但栓塞修复是需要提供能量和水分以恢复 木质部水分运输能力的生理代谢过程, 因此, 栓塞 修复并不是自发过程, 而需要一些生理活动促进水 分流向栓塞导管 (Zwieniecki \& Holbrook，2009; Secchi \& Zwieniecki, 2010, 2014)。长期以来人们一 直推测木质部栓塞修复是根压驱动的, 该过程同时 需要较低的蒸腾速率和较高的土壤有效水分, 从而 使木质部产生足以修复栓塞的正压(Zwieniecki \& Holbrook, 2009; Wheeler et al., 2013)。然而, 近期的 研究表明由于根压产生的正压力值普遍较低, 因此, 这种调节机制在草本植物中比较常见, 只有很少的 一部分木本被子植物利用根压修复栓塞, 且一般是 在冻融诱导的栓塞修复中(Brodersen \& McElrone, 2013)。近期的很多研究证实了在根压不存在的情况 下栓塞修复依然发生(Salleo et al., 2009; Knipfer et al., 2015, 2016)。此外, 高大木本植物在蒸腾活跃期, 其木质部栓塞甚至在木质部水柱处于负压时仍可修 复。以上结果表明: 根压驱动栓塞修复的机制在木 本植物中的作用有限(Zwieniecki \& Holbrook, 2009; Wheeler et al., 2013)。

近年来, 越来越多的实验提供了负压下木质部 栓塞修复的证据, 并提出了 “新的再充水” (novel refilling)栓塞修复机制(Hacke \& Sperry, 2003; Brodersen et al., 2010; Christman et al., 2012; Brodersen \& McElrone, 2013; Sperry, 2013; Zwieniecki et al., 2013; Rolland et al., 2015)。目前, 有关栓塞修复机 制虽然尚无完整的结论, 但对导管“新的再充水”过 程提出了不同的假说, 概括起来主要有4种: (1)渗透 调节假说; (2)反渗透调节假说; (3)韧皮部驱动再充 水假说; (4)韧皮部卸载假说(图1)。

\section{1 渗透调节假说}

渗透调节假说(the osmotic hypothesis)是指栓塞 导管再充水所需要的驱动力来自于栓塞导管壁上薄 液流层(thin sap layer)溶质浓度增加所产生的渗透 压, 即溶质浓度增加会降低栓塞导管的渗透势, 从 而引起水分在正压下流入栓塞导管(Ewers et al., 2001; Hacke \& Sperry, 2003); 其中的溶质来源于相 


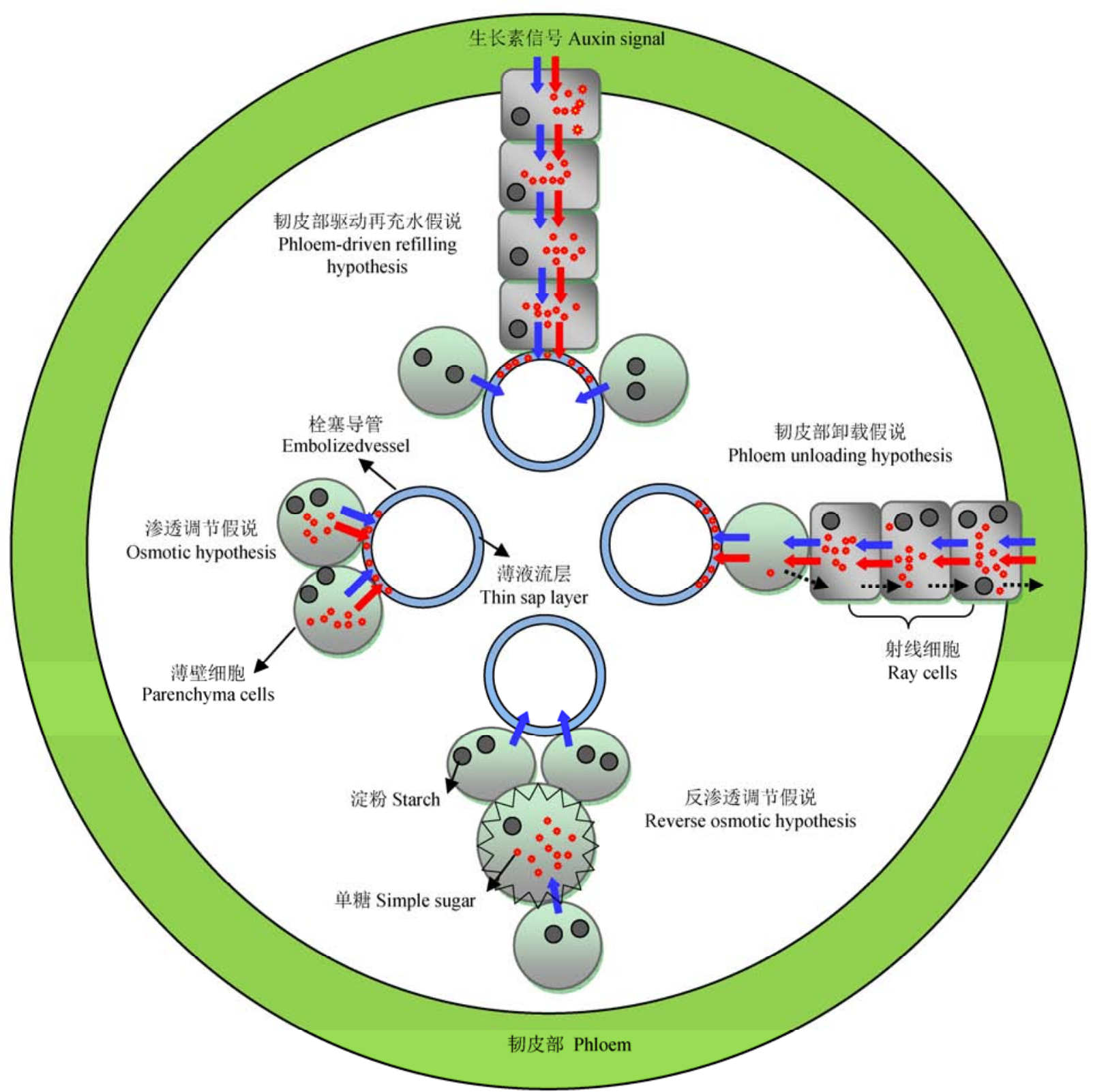

图1 木质部导管“新的再充水”栓塞修复机制的4种假说。图中红色和蓝色箭头分别代表溶质流和水流; 虚线箭头代表薄壁细 胞发出信号。

Fig. 1 Four hypotheses for the mechanisms of the novel refilling in xylem embolism repair. Red and blue arrows represent solutes and water flow, respectively; the dash arrows represent the signal from parenchyma cells.

邻薄壁细胞中无机离子和淀粉水解过程所产生的糖 等(Tyree et al., 1999; Bucci et al., 2003; Hacke \& Sperry, 2003; Améglio et al., 2004; Salleo et al., 2004)。关于渗透调节假说还有一种说法: 栓塞导管 周围薄壁细胞的纹孔膜本身可能起到半渗透屏障作 用, 阻止木质部树液中较大有机溶质(如蛋白质或 多糖)的进入, 允许渗透压的建立, 驱动水分流向栓 塞导管以完成再充水, 这种假说称为纹孔膜渗透假
说 (pit membrane osmosis hypothesis, Hacke \& Sperry, 2003; Salleo et al., 2004; Nardini et al., 2011)。

\section{2 反渗透调节假说}

反渗透调节假说(the reverse osmotic hypothesis) 是指木质部导管周围活的薄壁细胞中的淀粉水解成 糖将降低自身的渗透势, 使得水分沿着水势梯度进 入薄壁细胞, 使得细胞完全膨胀, 从而提高膨压, 
进而在薄壁细胞外部组织中产生组织压。这个组织 压会把水分从其他活细胞中挤出来, 并在正压力下 将水分挤进栓塞的导管, 使之再充水(Nardini et al., 2011)。反渗透调节假说只涉及向栓塞导管传递水 分, 而并未向栓塞导管输入溶质。然而, 对该假说存 在一些不同意见: 首先, Tyree等(1999)认为膨压产 生的组织压将向各个方向扩散, 不能驱动水分朝指 定的栓塞导管方向流动; 其次, 薄壁细胞通过质量 流既向内吸收水分以提高自身细胞膨压, 又向外把 水分传递到再充水的导管中(Comstock, 1999; Tyree et al., 1999), 这在热动力学上是不可能的, 似乎不 太现实, 还需要进一步验证。

\section{3 㓞皮部驱动再充水假说}

Salleo等(1996)基于树干环割对木质部再充水 过程的抑制作用(即环割对栓塞修复的抑制作用源 于㓞皮部正压的扩散), 首次提出了韧皮部在木质 部栓塞修复中的作用, 随后得到许多研究的证实 (Zwieniecki et al., 2000; Bucci et al., 2003; Salleo et al., 2006)。韧皮部驱动再充水假说 (the phloemdriven refilling hypothesis)是指在木质部发生栓塞 时, 从叶向茎运输的生长素信号激活了质子洜 (proton pump), 诱导㓞皮部装载溶质的增加, 从而 增加韧皮部的压力, 进而促使溶质沿射线薄壁细胞 进行径向运动, 进入栓塞的木质部导管, 降低水势, 促进更多的水分进入导管, 使木质部重新充水 (Salleo et al., 1996)。这种情况下, 㓞皮部被认为是 驱动水分径向流动所需要的正压力的来源。Salleo 等(2006)对月桂韧皮部在栓塞修复中可能作用的研 究表明: 㓞皮部输出信号使得木质部薄壁细胞中的 淀粉水解, 然后驱动糖流向栓塞导管, 之后水分沿 着渗透势流向栓塞导管以完成再充水; 但他们同时 指出有研究认为韧皮部的压力可能类似于第二种假 说中的 “组织压”, 通过环割作用使组织压失效, 再 通过外界施加径向压力模拟韧皮部的生理压力可使 其恢复。但这种说法仍存在争论, 需要进一步证实。

\section{4 韧皮部卸载假说}

韧皮部卸载假说(the phloem unloading hypothesis)指出, 在渗透调节驱动的木质部再充水过程中, 木质部薄壁细胞中的淀粉解聚成糖后, 被运输到空 穴化导管中, 产生再充水所需要的渗透驱动力; 相 反, 如果木质部薄壁细胞中淀粉含量不足, 将使这 些薄壁细胞变成很强的汇, 通过射线细胞反馈到㓞
皮部，驱使㓞皮部卸载糖和水分，通过射线细胞流 动到再充水导管(Nardini et al., 2011)。木质部薄壁细 胞中的碳水化合物储存库的消耗为韧皮部卸载创造 了一个强烈信号, 引发韧皮部中的糖和水通过木质 部射线进行径向移动(Salleo et al., 2009; Nardini et al., 2011; Brodersen et al., 2013)。这种栓塞修复方式 是渗透调节假说和㓞皮部驱动再充水假说的结合。 许多研究证实木质部栓塞形成过程中薄壁细胞中的 淀粉含量下降(Salleo et al., 2009; Secchi \& Zwieniecki, 2011); 栓塞形成与修复的循环过程也许会 耗尽这些碳水化合物的储存库, 使得再充水机制在 反复的干旱胁迫后失去作用。这种消耗将最终导致 植物碳饥饿, 从而使树木在持久或多次干旱胁迫事 件中难以恢复(McDowell et al., 2008)。

以上 4 种假说(除了纹孔膜渗透假说外)均需要 再充水导管 (正压)与张力状态下的功能性导管(负 压)保持水力隔离; 否则, 流入再充水导管中的水分 将立即被张力状态下的功能性导管吸出(Holbrook \& Zwieniecki, 1999; Salleo et al. 2004; Nardini et al., 2011)。纹孔在再充水导管与周围功能性导管的水力 隔离中起到阀门作用。这种水力隔离机制需要非零 的接触角度(即水滴与导管内壁之间的夹角)和向外 打开的具缘纹孔腔(Zwieniecki \& Holbrook, 2000)。 非零的接触角度意味着水分在导管内壁表面呈水珠 状增长, 而不是在导管内壁表面铺展开, 从而阻止 了进入气体填充导管的水分沿着细胞壁表面与功能 性导管连接。向外打开的具缘纹孔腔是在具缘纹孔 内形成气-水交界面的凸曲面。当水分进入具缘纹孔 的直壁通道时, 气-水交界面是凹的。在这种情况下 水稳态压力和表面张力在同一方向上, 把水分拉入 具缘纹孔。然而, 当弯曲面达到向外展开的纹孔膜 时, 由于导管壁的角度发生变化将导致气-水交界 面变成凸曲面, 凸曲面意味着表面张力将抵抗再充 水导管腔内的水稳态压力, 从而完成了水力隔离 (Holbrook \& Zwieniecki, 1999)。

对水分胁迫植物的基因表达分析发现, 在再充 水过程中离子运输、水通道蛋白和碳代谢具有上调 作用(Secchi et al., 2011; Secchi \& Zwieniecki, 2012)。 Perrone等(2012)研究葡萄树叶柄的再充水过程中发 现相似的模式, 即碳代谢和水通道蛋白表达均强烈 上调。碳水化合物代谢的上调可促进蔗糖的释放; 这些蔗糖被运输到栓塞导管而产生渗透梯度, 在水 
通道蛋白(质膜内在蛋白(PIP) 和液泡膜内在蛋白 (TIP)) 的上调作用下驱动水分进入栓塞导管 (Brodersen \& McElrone, 2013)。再充水过程中水通 道蛋白的强烈上调增加了细胞膜活性, 进而增加了 膜的水力导度, 促进了水分的释放, 减少了栓塞修 复时间(Nardini et al., 2011; Secchi et al., 2011)。此 外, 木本植物中水通道蛋白(PIPs和TIPs)的基因表 达随栓塞及其修复的动态过程而变化(Secchi et al., 2011; Perrone et al., 2012; Laur \& Hacke, 2013; Pou et al., 2013)。Secchi等(2011)研究Populus trichocarpa 茎栓塞再充水过程中的基因活性, 表明不同的水通 道蛋白亚族在再充水过程中强烈上调, 这种上调作 用也许促进了水分的释放以使得栓塞导管再充水。

Secchi和Zwieniecki (2010)研究表明: PIP2亚族的表 达对栓塞胁迫没有变化; 而PIP1基因表达在响应于 栓塞时上调, 在再充水后立刻下调。尽管水通道蛋 白促进薄壁细胞和木质部之间的水分交流, 但水通 道蛋白是被动的运输者, 使得水分通过他们沿着能 量梯度流动(Secchi et al., 2011; Secchi \& Zwieniecki, 2012)。综上所述, 导管“新的再充水”栓塞修复过程 是一个需要能量代谢和精细协调的生理学过程, 涉 及淀粉水解、溶质和水分的运输。

\section{4 针叶树种和被子植物树种木质部栓塞修 复的差异性}

栓塞修复机制中的再充水过程是基于木质部薄 壁细胞中淀粉水解成可溶性糖, 产生再充水所需要 的驱动力, 驱使渗透水分流向栓塞导管, 引起水分 被动地沿着渗透势梯度移动 (Zwieniecki \& Holbrook, 2009; Nardini et al., 2011), 而且韧皮部中的 淀粉卸载也可促进栓塞的修复。可见, 非结构性碳 水化合物可通过渗透调节和维持细胞膨胀在栓塞修 复过程中起到关键作用。糖最有可能来源于㓞皮部 或薄壁组织细胞中非结构性碳水化合物的水解 (Canny, 1993)。植物由于导管至㓞皮部的距离随边 材深度而变, 因此可以通过将薄壁组织嵌入贯穿整 个木质部, 从而使更多导管在栓塞时能接触到糖 (Johnson et al., 2012)。因此, 植物木质部栓塞修复能 力的差异性主要与其薄壁组织细胞数量和非结构性 碳水化合物浓度有关(Salleo et al., 2009; Nardini et al., 2011; Johnson et al., 2012; Secchi \& Zwieniecki, 2012)。
针叶树种和被子植物树种木质部输水结构的不 同, 使其水分运输效率和抗旱能力差异显著 (McCulloh et al., 2010)。首先, 针叶树种依靠结构简 单且直径较小的管胞输导水分; 而被子植物依靠结 构复杂且直径较大的导管进行水分运输; 这些内部 结构特性差异使两种功能群植物在应对水分胁迫时 采取不同的避免或容忍对策(Carnicer et al., 2013)。 木本被子植物又分为环孔材和散孔材, 两者解剖结 构的差异也会导致其栓塞修复能力存在差异。与导 管直径分布均匀的散孔材相比, 环孔材产生直径大 小不同的导管对栓塞修复更有利。这种对策可确保 易空穴化的大导管与抗空穴化的小导管距离较近, 作为水分储存库有利于再充水或缓解干旱诱导的木 质部张力(Davis et al., 1999; Pittermann \& Sperry, 2003)。针叶树种茎干通常经历栓塞的频率较低, 但 其末端器官(叶和根)栓塞发生和修复的频率较高; 相反，木本被子植物的茎干经历栓塞和修复频繁， 而其末端器官经历栓塞的频率较低。这种差异性可 能与两者的修复栓塞能力有关(Johnson et al., 2009, 2012; Meinzer et al., 2009; Choat et al. 2012)。就茎干 而言, 针叶树种茎干的木质部薄壁组织比被子植 物少, 其非结构性碳水化合物浓度较低, 从而其栓 塞修复能力较差, 因此针叶树种构建了较大的水力 安全阈值, 以阻止栓塞发生(Panshin \& Zeeuw, 1980)。然而, 针叶树种的末端组织(叶和根)栓塞频 繁地发生且很容易修复, 可能是其末端器官中存在 丰富的薄壁组织细胞。针叶树种叶组织中的管胞周 围是传输组织(传输组织是管胞和活薄壁组织的结 合, 可以是中空的且在低压下不会产生空穴化), 可 提供水分给其余的管胞或缓解内部压力梯度 (Johnson et al., 2009)。此外, 早期研究表明, 针叶树 种叶片中的传输组织也可作为再充水所需要的糖的 来源(Canny, 1993)。

其次, 由于纹孔膜结构的差异, 针叶树种和木 本被子植物的水力隔离机制可能不同(Brodribb et al., 2012)。针叶树种纹孔膜中有纹孔塞结构, 充水 的管胞通过吸入纹孔膜, 使得纹孔塞堵住纹孔口, 与栓塞管胞产生水力隔离, 阻止了气体的进一步扩 展(Sperry \& Tyree, 1990; Choat et al., 2008; Cochard et al., 2009)。相反, 木本被子植物的纹孔膜中虽然 没有纹孔塞, 但有均一的多微孔结构, 可通过毛细 管力堵住纹孔口, 防止栓塞扩张(Pittermann et al., 
2005)。那么, 针叶树种的纹孔塞结构是否优于木本 被子植物的纹孔膜结构? 研究表明两者空穴化阻力 的范围相同, 即针叶树种的纹孔塞和木本被子植物 的纹孔膜起到同样的阻碍气种扩张的作用 (Pittermann et al., 2005)。尽管木本被子植物纹孔膜 具有较高的水力阻力, 但其同质纹孔膜结构能够有 规律地修复栓塞导管(Sperry et al., 1987; Salleo et al., 2004; Brodersen et al., 2010), 潜在的抵抗空穴化 疲劳引起的机械损害, 同时起到水力隔离作用(Hacke et al., 2001; Zwieniecki \& Holbrook, 2009)。相反, 如果 针叶树种的纹孔塞被吸走且远离纹孔口, 纹孔膜塞 缘的微纤丝束的拉伸和机械损坏不能被修复, 管胞 的栓塞则不能被修复(Sperry \& Tyree, 1990)。

\section{5 木质部栓塞阻力和修复能力的权衡关系}

既然植物木质部导管栓塞形成主要是与木材理 化性质有关的物理过程, 而栓塞导管再充水修复是 消耗能量的生理代谢过程(Salleo et al., 2004; Secchi \& Zwieniecki, 2011), 那么木质部栓塞阻力与修复 能力是否存在一种权衡关系呢? 对于空穴化脆弱的 物种, 其低的空穴化阻力是否可通过高的木质部恢 复能力补偿? 木质部空穴化阻力用 $P_{50}$ 表示, 是指 水力导度损失 $50 \%$ 对应的木质部水势; 而木质部恢 复能力用木质部恢复指数 (xylem recovery index, $X R I$ ) 表示, 是指对植物进行干旱胁迫处理(胁迫程度 达到其木质部水势接近 $P_{50}$ 的水平), 然后复水 $12 \mathrm{~h}$, 此时的木质部导水率与自然条件下木质部最大导水 率之比即为木质部恢复指数(Ogasa et al., 2013)。 Ogasa等(2013)研究了7个温带木本植物发现, XRI因 物种而异, 不同物种的空穴化阻力 $\left(\left|P_{50}\right|\right)$ 与 $X R I$ 负相 关, 表明不同物种木质部空穴化脆弱性与木质部栓 塞修复能力具有一定的权衡关系, 即空穴化脆弱的 物种通过高的木质部恢复能力进行补偿; 但对于抗 空穴化阻力强的树种是否具有相似规律尚不清楚。

木材密度可能是木质部空穴化阻力和恢复能力 权衡关系的调节因子(Ogasa et al., 2013)。木材密度 与空穴阻力正相关、与XRI负相关, 表明空穴化阻力 和木质部恢复表现之间的协调性与木材功能特性相 关(Ogasa et al., 2013)。木材密度较大的木质部抵抗 空穴化能力较强, 而木材密度较低的木质部, 储水 能力较高, 有利于木质部再充水进行栓塞修复。这
些发现解释了生长在多变环境条件下的物种为何能 够长期维持水分运输。此外, 从生物量分配角度看, 物种的木材密度和生长速率之间也存在一定的权衡 关系(Chave et al., 2009, Poorter et al., 2010), 从而对 植物结构和功能产生限制(Ishida et al., 2008), 其中 对木材密度的限制会影响空穴化阻力和木质部恢复 表现的权衡关系。木质部空穴化阻力和恢复表现之 间的权衡关系在发育上是否独立尚需要在更多的木 本植物上加以证实(Ackerly \& Reich, 1999; Maherali et al. 2006)。

\section{6 优先研究问题}

\section{1 改进木质部栓塞测定技术避免人为影响}

目前关于木本植物木质部栓塞发生及修复过程 的分歧很大程度上源于技术的不确定性。在实验操 作过程中, 由于木质部内存在的张力使得在切割样 本时很容易人为诱导栓塞的发生, 因此, 很难准确 量化栓塞形成和修复的过程。鉴于此, 研究者首先 应重新考虑现有数据, 改进空穴化阻力的测量方 法。在使用离体法测量木质部栓塞时, 应尽量避免 在自然张力下切割样本诱导栓塞发生的人为影响; 且又要避免通过水化释放样本张力而发生再充水过 程导致栓塞修复的人为影响。一般情况下, 在第一 次剪切使样本离体后, 要在水下对末端切口进行二 次修剪, 这样可确保在第一次剪切过程中可能被吸 收进木质部导管的微气泡被去除。而Wheeler等 (2013)所描述的张力下切割诱导的人为影响可能是 由于其第一次剪切时的取样长度精确到测量时的样 本长度, 没有留出足够的长度以便第二次修剪时去 掉微气泡可能存在的距离。Venturas等(2015)的研究 表明再次修剪时, 切割掉0.5-1.5 $\mathrm{cm}$ 的木质部长度 可去除进入的微气泡, 避免张力状态下剪切诱导的 人为影响, 所以在最初取样时, 样本长度要比测量 长度稍微长一点(1-3 cm左右)。而为了避免再充水 过程导致栓塞修复的人为影响, 尽量避免使用水化 释放张力的方法, 即使使用也应该慎重, 比如水化 时间不要过长; 或通过环割、化学处理抑制再充水 过程。其次, 为了弥补离体法测量栓塞的缺陷, 研究 者应尽量使用无损可视化技术检测栓塞形成及修复 过程, 如核磁共振成像技术, X射线显微层析技术等 (Zwieniecki et al., 2013; Cochard et al., 2014)。 


\section{2 验证“新的再充水”栓塞修复机制假说及引发 木质部再充水的信号}

由于木质部栓塞形成位置的空间随机性，所以 植物感知栓塞的能力在生理上非常重要。只有植物 真正需要修复栓塞时, 再充水过程才需要被激活, 这意味着需要一个信号响应路径(signal-to-response pathway)以察觉空穴化发生及之后的栓塞修复事件 (Zwiehiecki \& Holbrook, 2009)。首先, 发生空穴事 件产生机械信号(如直接的导管壁振动, 或间接的 导管周围活细胞的膨压变化); 其次, 机械信号导致 细胞溶质中 $\mathrm{Ca}^{2+}$ 浓度的变化, 引发信号传递, 导致 淀粉解聚成糖, 在质子泵和糖 $/ \mathrm{H}^{+}$转运蛋白共同作 用下运载到栓塞导管, 产生再充水所需要的驱动 力; 然后, 从导管周围薄壁细胞运出的糖将促使这 些细胞变成汇, 发出促进韧皮部卸载糖和水分的信 号, 流向导管周围的活细胞和栓塞导管(Nardini et al., 2011)。相反, 一些研究认为木质部导管的空穴 化事件不足以产生引发再充水的机械信号, 也不能 提供植物结束再充水过程的关闭信号 (Secchi \& Zwieniecki, 2011)。有学者提出木质部薄壁细胞不断 地释放少量蔗糖到木质部导管, 蔗糖将沿着功能性 导管的蒸腾流不断地被运走。当导管栓塞后, 活细 胞将继续释放糖到栓塞导管的薄液流层, 但在栓塞 情况下, 蔗糖不能被蒸滕流运走, 因此建立了高的 糖浓度。质外体糖浓度的增加将作为信号引发栓塞 修复。修复后的导管将再次与蒸腾流连接, 糖逐步 溶解将提供关闭再充水机制的信号。鉴于这种不确 定性, 需要进一步开展栓塞和引发再充水过程的实 验验证研究, 以证实木质部新的再充水栓塞修复机 制。

\section{3 阐明木质部栓塞与修复特性的树种间差异及 其可能的权衡关系}

Ogasa等(2013)的研究发现了空穴化阻力与木 质部修复能力之间存在权衡关系。这种权衡关系是 通过对照的木材功能特性的调节, 如密度大、抗空 穴化的木质部vs. 密度小、可恢复的木质部。这一发 现有助于解释长期维持水分运输的物种空穴化脆弱 性变异性大的原因。此外, 木质部导管大小的均匀 性也可影响导管“新的再充水”机制。与导管大小一 致的树种相比, 产生不同大小的导管是有利的。这 种对策可使大直径、易空穴化的导管与小直径、抗 空穴化的导管位置相邻, 从而空穴化阻力强的导管
可以作为储水库, 以帮助栓塞导管的再充水或缓解 干旱诱导的木质部张力 (Brodersen \& McElrone, 2013)。尽管如此, 栓塞修复特性还不完全清楚, 尚 不清楚“新的再充水”修复机制是否限制于某些特定 物种、器官, 甚至木质部内特定的地点。此外, 同一 植物不同器官(根系、树干、茎、叶柄和叶脉)中的 木质部“新的再充水”的程度是否一样也尚未可知? 这些器官木质部解剖结构和在植物体中位置的差异 可能会影响再充水机制, 如: 根系的栓塞修复可能 受根压驱动, 而在冠层较高位置的器官可能更依赖 于“新的再充水”机制修复栓塞。今后的工作仍需要 评价不同植物及同一植物不同器官中“新的再充水” 栓塞修复机理及栓塞阻力与恢复能力之间的权衡关 系。

\section{4 加强碳代谢和水通道蛋白表达与木质部栓塞 及其修复关系的生理生化研究}

在木质部栓塞修复的再充水过程中, 碳代谢和 水通道蛋白表达起到重要作用(Brodersen \& McElrone, 2013)。碳水化合物代谢的增加可促进糖的释 放, 为再充水提供能源。糖作为渗透剂转移到邻近 的栓塞导管, 建立渗透梯度, 驱动水分流入栓塞导 管, 因此非结构性碳水化合物是植物抵抗干旱诱导 栓塞的重要特性, 可提高植物对水分亏缺的容忍度 (O’Brien et al., 2014)。但非结构性碳水化合物储量 与栓塞修复能力的关系尚不清楚。此外, 水通道蛋 白表达增加或活性增加, 可促进更多的水分进入栓 塞导管, 使木质部栓塞修复。薄壁细胞中水通道蛋 白的表达和激活, 可促进韧皮部的卸载。尤其当韧 皮部卸载伴随着糖进入栓塞木质部导管的过程, 在 韧皮部到木质部的路径上, 水通道蛋白的上调作用 是必要条件; 这样可增加径向的水力导度, 显著缩 短栓塞再充水需要的时间(Nardini et al., 2011)。然 而, 在再充水过程中, 水通道蛋白家族的哪个成员 在修复过程起到关键作用、水通道蛋白的激活和表 达的精确位置在哪儿, 尚未可知(冷华妮, 2012)。今 后木质部栓塞修复机理研究需要探索水分运输路径 上发生的化学和基因水平上的变化, 通过对再充水 过程中水通道蛋白的定位可加深对栓塞修复过程的 理解, 阐明栓塞再充水过程中主要的水流路径和最 终的水流方向(Nardini et al., 2011; Brodersen \& McElrone, 2013); 同时还应强化非结构性碳水化合 物浓度、淀粉和糖的比例及其在根、茎、叶器官上 
的分配与栓塞修复之间关系的研究, 这对笁选生态 策略上耐旱造林树种具有重要意义。

基金项目 教育部长江学者和创新团队发展计划 (IRT_15R09)和中央高校基本科研业务费专项资金 项目(2572015AA16)。

\section{参考文献}

Ackerly DD, Reich PB (1999). Convergence and correlations among leaf size and function in seed plants: A comparative test using independent contrasts. American Journal of Botany, 86, 1272-1281.

Améglio T, Decourteix M, Alves G, Valentin V, Sakr S, Julien JL, Petel G, Guilliot A, Lacointe A (2004). Temperature effects on xylem sap osmolarity in walnut trees: Evidence for a vitalistic model of winter embolism repair. Tree Physiology, 24, 785-793.

Brodersen CR, McElrone AJ (2013). Maintenance of xylem network transport capacity: A review of embolism repair in vascular plants. Frontiers in Plant Science, 4, 1-11.

Brodersen CR, McElrone AJ, Choat B, Lee EF, Shackel KA, Matthews MA (2013). In vivo visualizations of droughtinduced embolism spread in Vitis vinifera. Plant Physiology, 161, 1820-1829.

Brodersen CR, McElrone AJ, Choat B, Matthews MA, Shackel KA (2010). The dynamics of embolism repair in xylem: In vivo visualizations using high-resolution computed tomography. Plant Physiology, 154, 1088-1095.

Brodribb TJ, Bowman DJMS, Nichols S, Delzon S, Burlett R (2010). Xylem function and growth rate interact to determine recovery rates after exposure to extreme water deficit. New Phytologist, 188, 533-542.

Brodribb TJ, Cochard H (2009). Hydraulic failure defines the recovery and point of death in water-stressed conifers. Plant Physiology, 149, 575-584.

Brodribb TJ, Pittermann J, Coomes DA (2012). Elegance versus speed: Examining the competition between conifer and angiosperm trees. International Journal of Plant Sciences, 173, 673-694.

Bucci SJ, Scholz FG, Goldstein G, Meinzer FC, Sternberg LDASL (2003). Dynamic changes in hydraulic conductivity in petioles of two savanna tree species: Factors and mechanisms contributing to the refilling of embolized vessels, Plant, Cell \& Environment, 26, 1633-1645.

Canny MJ (1993). Transfusion tissue of pine needles as a site of retrieval of solutes from the transpiration stream. New Phytologist, 123, 227-232.

Carnicer J, Barbeta A, Sperlich D, Coll M, Penuelas J (2013). Contrasting trait syndromes in angiosperms and conifers are associated with different responses of tree growth to temperature on a large scale. Front Plant Science, 4, 409.

Chave J, Coomes D, Jansen S, Lewis SL, Swenson NG, Zanne AE (2009). Towards a worldwide wood economics spec- trum. Ecology Letters, 12, 351-366.

Choat B, Cobb AR, Jansen S (2008). Structure and function of bordered pits: New discoveries and impacts on whole-plant hydraulic function. New Phytologist, 177, 608-626.

Choat B, Jansen S, Brodribb TJ, Cochard H, Delzon S, Bhaskar R, Bucci SJ, Feild TS, Gleason SM, Hacke UG, Jacobsen AL, Lens F, Maherali H, Martinez-Vilalta JM, Mayr S, Mencuccini M, Mitchell PJ, Nardini A, Pittermann J, Pratt RB, Sperry JS, Westoby M, Wright IJ, Zanne AE (2012). Global convergence in the vulnerability of forests to drought. Nature, 491, 752-755.

Choat B, Lahr EC, Melcher PJ, Zwieniecki MA, Holbrook NM (2005). The spatial pattern of air seeding thresholds in mature sugar maple trees. Plant, Cell \& Environment, 28, 1082-1089.

Christman MA, Sperry JS, Smith DD (2012). Rare pits, large vessels and extreme vulnerability to cavitation in a ring-porous tree species. New Phytologist, 193, 713-720.

Cochard H, Badel E, Herbette S, Delzon S, Choat B, Jansen S (2013). Methods for measuring plant vulnerability to cavitation: A critical review. Journal of Experimental Botany, ert193.

Cochard H, Delzon S (2013). Hydraulic failure and repair are not routine in trees. Annals of Forest Science, 70, 659-661.

Cochard H, Delzon S, Badel E (2014). X-ray microtomography (micro-CT): A reference technology for high-resolution quantification of xylem embolism in trees. Plant, Cell \& Environment, 38, 201-206.

Cochard H, Holtta T, Herbette S, Delzon S, Mencuccini M (2009). New insights into the mechanisms of water-stressinduced cavitation in conifers. Plant Physiology, 151, 949-954.

Comstock JP (1999). Why Canny's theory doesn't hold water. American Journal of Botany, 86, 1077-1081.

Davis SD, Sperry JS, Hacke UG (1999). The relationship between xylem conduit diameter and cavitation caused by freezing. American Journal of Botany, 86, 1367-1372.

Ewers FW, Améglio T, Cochard H, Beaujard F, Martignac M, Vandame M, Bodet C, Cruiziat AP (2001). Seasonal variation in xylem pressure of walnut trees: Root and stem pressures. Tree Physiology, 21, 1123-1132.

Hacke U, Sperry JS (2003). Limits to xylem refilling under negative pressure in Laurus nobilis and Acer negundo. Plant, Cell \& Environment, 26, 303-311.

Hacke UG, Stiller V, Sperry JS, Pittermann J, McCulloh KA (2001). Cavitation fatigue. Embolism and refilling cycles can weaken the cavitation resistance of xylem. Plant Physiology, 125, 779-786.

Holbrook NM, Ahrens ET, Burns MJ, Zwieniecki MA (2001). In vivo observation of cavitation and embolism repair using magnetic resonance imaging. Plant Physiology, 126, 
27-31.

Holbrook NM, Zwieniecki MA (1999). Embolism repair and xylem tension: Do we need a miracle? Plant Physiology, 120, 7-10.

Hölttä T, Vesala T, Perämäki M, Nikinmaa E (2006). Refilling of embolised conduits as a consequence of 'Munch water' circulation. Functional Plant Biology, 33, 949-959.

Ishida A, Nakano T, Yazaki K, Matsuki S, Koike N, Lauenstein DL, Shimizu M, Yamashita N (2008). Coordination between leaf and stem traits related to leaf carbon gain and hydraulics across 32 drought-tolerant angiosperms. Oecologia, 156, 193-202.

Jansen S, Choat B, Pletsers A (2009). Morphological variation of intervessel pit membranes and implications to xylem function in angiosperms. American Journal of Botany, 96, 409-419.

Johnson DM, McCulloh KA, Woodruff DR, Meinzer FC (2012). Hydraulic safety margins and embolism reversal in stems and leaves: Why are conifers and angiosperms so different? Plant Science, 195, 48-53.

Johnson DM, Woodruff DR, McCulloh KA, Meinzer FC (2009). Leaf hydraulic conductance, measured in situ, declines and recovers daily: Leaf hydraulics, water potential and stomatal conductance in four temperate and three tropical tree species. Tree Physiology, 29, 879-887.

Knipfer T, Cuneo I, Brodersen C, McElrone AJ (2016). In-situ visualization of the dynamics in xylem embolism formation and removal in the absence of root pressure: A study on excised grapevine stems. Plant Physiology, doi: 10.1104/pp.16.00136.

Knipfer T, Eustis A, Brodersen C, Walker AM, McElrone AJ (2015). Grapevine species from varied native habitats exhibit differences in embolism formation/repair associated with leaf gas exchange and root pressure. Plant, Cell \& Environment, 38, 1503-1513.

Laur J, Hacke UG (2013). Transpirational demand affects aquaporin expression in poplar roots. Journal of Experimental Botany, 64, 2283-2293.

Leng HN (2012). Cloning and Expression of PIPs Gene in Populus and Aquaporins Role in Embolism Recovery. $\mathrm{PhD}$ dissertation, Chinese Academy of Forestry, Beijing. (in Chinese). [冷华妮 (2012). 植物栓塞修复机制与质膜内 在水通道蛋白基因的克隆、表达和转基因研究. 博士学 位论文, 中国林业科学研究院, 北京.]

Li R, Jiang ZM, Zhang SX, Cai J (2015). A review of new research progress on the vulnerability of xylem embolism of woody plants. Chinese Journal of Plant Ecology, 39, 838-848. (in Chinese with English abstract) [李荣, 姜在 民, 张硕新, 蔡靖 (2015). 木本植物木质部栓塞脆弱性 研究新进展. 植物生态学报, 39, 838-848.]

Maherali H, Moura CF, Caldeira MC, Willson CJ, Jackson RB (2006). Functional coordination between leaf gas exchange and vulnerability to xylem cavitation in temperate forest trees. Plant, Cell \& Environment, 29, 571-583.

McCulloh K, Sperry JS, Lachenbruch B, Meinzer FC, Reich PB (2010). Moving water well: Comparing hydraulic efficiency in twigs and trunks of coniferous, ring-porous, and diffuse-porous saplings from temperate and tropical forests. New Phytologist, 186, 439-450.

McCulloh KA, Meinzer FC (2015). Further evidence that some plants can lose and regain hydraulic function daily. Tree Physiology, 35, 691-693.

McDowell N, Pockman WT, Allen CD, Breshears DD, Cobb N, Kolb T, Plaut J, Sperry J, West A, Williams DG, Yepez EA (2008). Mechanisms of plant survival and mortality during drought: Why do some plants survive while others succumb to drought? New Phytologist, 178, 719-739.

McElrone AJ, Brodersen CR, Alsina MM, Drayton WM, Matthews MA, Shackel KA, Wada H, Zufferey V, Choat B (2012). Centrifuge technique consistently overestimates vulnerability to water stress-induced cavitation in grapevines as confirmed with high-resolution computed tomography. New Phytologist, 196, 661-665.

Meinzer FC, Johnson DM, Lachenbruch B, McCulloh KA, Woodruff DR (2009). Xylem hydraulic safety margins in woody plants: Coordination of stomatal control of xylem tension with hydraulic capacitance. Functional Ecology, 23, 922-930.

Meinzer FC, McCulloh KA (2013). Xylem recovery from drought-induced embolism: Where is the hydraulic point of no return? Tree Physiology, 33, 331-334.

Nardini A, Lo Gullo MA, Salleo S (2011). Refilling embolized xylem conduits: Is it a matter of phloem unloading? Plant Science, 180, 604-611.

O’Brien MJ, Leuzinger S, Philipson CD, Tay J, Hector A (2014). Drought survival of tropical tree seedlings enhanced by non-structural carbohydrate levels. Nature Climate Change, 4, 710-714.

Ogasa M, Miki NH, Murakami Y, Yoshikawa K (2013). Recovery performance in xylem hydraulic conductivity is correlated with cavitation resistance for temperate deciduous tree species. Tree Physiology, 33, 335-344.

Panshin AJ, Zeeuw CD (1980). Textbook of Wood Technology. McGraw-Hill, New York.

Perrone I, Pagliarani C, Lovisolo C, Chitarra W, Roman F, Schu-bert A (2012). Recovery from water stress affects grape leaf petiole transcriptome. Planta, 235, 1383-1396.

Pittermann J, Sperry J (2003). Tracheid diameter is the key trait determining the extent of freezing-induced embolism in conifers. Tree Physiology, 23, 907-914.

Pittermann J, Sperry JS, Hacke UG, Wheeler JK, Sikkema EH (2005). Torus-margo pits help conifers compete with angiosperms. Science, 310, 1924-1924.

Plavcová L, Jansen S, Klepsch M, Hacke U (2012). Nobody’s perfect: Can irregularities in pit structure influence 
vulnerability to cavitation? Frontiers in Plant Science, 4, 453-453.

Poorter L, McDonald I, Alarcón A, Fichtler E, Licona JC, Peña-Claros M, Sterck F, Villegas Z, Sass-Klaassen U (2010). The importance of wood traits and hydraulic conductance for the performance and life history strategies of 42 rainforest tree species. New Phytologist, 185, 481-492.

Pou A, Medrano H, Flexas J, Tyerman SD (2013). A putative role for TIP and PIP aquaporins in dynamics of leaf hydraulic and stomatal conductances in grapevine under water stress and re-watering. Plant, Cell \& Environment, 36, 828-843.

Rolland V, Dana MB, Lenné T, Bryant G, Chen H, Wolfe J, Holbrook NM, Stanton DE, Ball MC (2015). Easy come, easy go: Capillary forces enable rapid refilling of embolized primary xylem vessels. Plant Physiology, 168, 1636-1647.

Salleo S, Lo Gullo MA, de Paoli D, Zippo M (1996). Xylem recovery from cavitation-induced embolism in young plants of Laurus nobilis: A possibile mechanism. New Phytologist, 132, 47-56.

Salleo S, Lo Gullo MA, Trifilò P, Nardini A (2004). New evidence for a role of vessel-associated cells and phloem in the rapid xylem refilling of cavitated stems of Laurus nobilis L. Plant, Cell \& Environment, 27, 1065-1076.

Salleo S, Nardini A, Pitt F, Lo Gullo MA (2000). Xylem cavitation and hydraulic control of stomatal conductance in laurel (Laurus nobilis L.). Plant, Cell \& Environment, 23, 71-79.

Salleo S, Trifilò P, Esposito S, Nardini A, Lo Gullo MA (2009). Starch-to-sugar conversion in wood parenchyma of field-growing Laurus nobilis plants: A component of the signal pathway for embolism repair? Functional Plant Biology, 36, 815-825.

Salleo S, Trifilò P, Lo Gullo MA (2006). Phloem as a possible major determinant of rapid cavitation reversal in stems of Laurus nobilis (laurel). Functional Plant Biology, 33, 1063-1074.

Scheenen TWJ, Vergeldt FJ, Heemskerk AM, van As H (2007). Intact plant magnetic resonance imaging to study dynamics in long-distance sap flow and flow-conducting surface area. Plant Physiology, 144, 1157-1165.

Scoffoni C, Sack L (2014). Are leaves "freewheelin”? Testing for a Wheeler-type effect in leaf xylem hydraulic decline. Plant, Cell \& Environment, 38, 534-543.

Secchi F, Gilbert ME, Zwieniecki MA (2011). Transcriptome response to embolism formation in stems of Populus trichocarpa provides insight into signaling and the biology of refilling. Plant Physiology, 157, 1419-1429.

Secchi F, Zwieniecki MA (2010). Patterns of PIP gene expression in Populus trichocarpa during recovery from xylem embolism suggest a major role for the PIP1 aquaporin subfamily as moderators of refilling process. Plant, Cell \&
Environment, 33, 1285-1297.

Secchi F, Zwieniecki MA (2011). Sensing embolism in xylem vessels: The role of sucrose as a trigger for refilling. Plant, Cell \& Environment, 34, 514-524.

Secchi F, Zwieniecki MA (2012). Analysis of xylem sap from functional (nonembolized) and nonfunctional (embolized) vessels of Populus nigra: Chemistry of refilling. Plant Physiology, 160, 955-964.

Secchi F, Zwieniecki MA (2014). Down-regulation of plasma intrinsic protein1 aquaporin in poplar trees is detrimental to recovery from embolism. Plant Physiology, 164, 1789-1799.

Sperry JS (2013). Cutting-edge research or cutting-edge artefact? An overdue control experiment complicates the xylem refilling story. Plant, Cell \& Environment, 11, 35-40.

Sperry JS, Donnelly JR, Tyree MT (1988). A method for measuring hydraulic conductivity and embolism in xylem. Plant, Cell \& Environment, 11, 35-45.

Sperry JS, Hacke UG (2004). Analysis of circular bordered pit function I. Angiosperm vessels with homogenous pit membranes. American Journal of Botany, 91, 369-385.

Sperry JS, Holbrook NM, Zimmermann MH, Tyree MT (1987). Spring filling of xylem vessels in wild grapevine. Plant Physiology, 83, 414-417.

Sperry JS, Tyree MT (1990). Water-stress-induced xylem embolism in three species of conifers. Plant, Cell \& Environment, 13, 427-436.

Stiller V, Sperry JS (2002). Cavitation fatigue and its reversal in sunflower (Helianthus annuus L.). Journal of Experimental Botany, 53, 1155-1161.

Torres-Ruiz JM, Jansen S, Choat B, McElrone AJ, Cochard H, Brodribb TJ, Badel E, Burlett R, Bouche PS, Brodersen CR, Li S, Morris H, Delzon S (2015). Direct X-ray microtomography observation confirms the induction of embolism upon xylem cutting under tension. Plant Physiology, 167, 40-43.

Trifilò P, Nardini A, Lo Gullo MA, Barbera PM, Savi T, Raimondo F (2015). Diurnal changes in embolism rate in nine dry forest trees: Relationships with species-specific xylem vulnerability, hydraulic strategy and wood traits. Tree Physiology, 35, 694-705.

Trifilò P, Raimondo F, Lo Gullo MA, Barbera PM, Salleo S, Nardini A (2014). Relax and refill: Xylem rehydration prior to hydraulic measurements favours embolism repair in stems and generates artificially low PLC values. Plant, Cell \& Environment, 37, 2491-2499.

Tyree MT, Salleo S, Nardini A, Lo Gullo MA, Mosca R (1999). Refilling of embolized vessels in young stems of laurel. Do we need a new paradigm? Plant Physiology, 120, 11-21.

Urli M, Porté A, Cochard H, Guengant Y, Burlett R, Delzon S (2013). Xylem embolism threshold for catastrophic hydraulic failure in angiosperm trees. Tree Physiology, 33, 
672-683.

Venturas MD, Mackinnon ED, Jacobsen AL, Pratt RB (2015). Excising stem samples underwater at native tension does not induce xylem cavitation. Plant, Cell \& Environment, 38, 1060-1068.

Vesala T, Hölttä T, Perämäki M, Nikinmaa E (2003). Refilling of a hydraulically isolated embolized xylem vessel: Model calculations. Annuals of Botany, 91, 419-428.

Wheeler JK, Huggett BA, Tofte AN, Rockwell FE, Holbrook NM (2013). Cutting xylem under tension or supersaturated with gas can generate PLC and the appearance of rapid recovery from embolism. Plant, Cell \& Environment, 36, 1938-1949.

Zimmermann MH (1983). Xylem Structure and the Ascent of Sap. Springer-Verlag, Berlin.

Zwieniecki MA, Holbrook NM (1998). Diurnal variation in xylem hydraulic conductivity in white ash (Fraxinus americana L.) red maple (Acer rubrum L.) and red spruce (Picea rubens Sarg.). Plant, Cell \& Environment, 21,
1173-1180.

Zwieniecki MA, Holbrook NM (2000). Bordered pit structure and vessel wall surface properties. Implications for embolism repair. Plant Physiology, 123, 1015-1020.

Zwieniecki MA, Holbrook NM (2009). Confronting Maxwell's demon: Biophysics of xylem embolism repair. Trends in Plant Science, 14, 530-534.

Zwieniecki MA, Hutyra L, Thompson MV, Holbrook NM (2000). Dynamic changes in petiole specific conductivity in red maple (Acer rubrum L.) tulip tree (Liriodendron tulipifera L.) and northern fox grape (Vitis labrusca L.). Plant, Cell \& Environment, 23, 407-414.

Zwieniecki MA, Melcher PJ, Ahrens ET (2013). Analysis of spatial and temporal dynamics of xylem refilling in Acer rubrum L. using magnetic resonance imaging. Frontiers in Plant Science, 4, 265.

特邀编委: 杜 盛 责任编辑: 李 敏

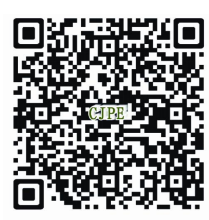

植物生态学报官网

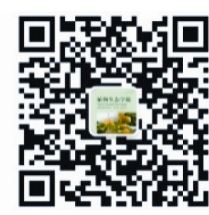

微信订阅号

期刊及学科

相关信息发布

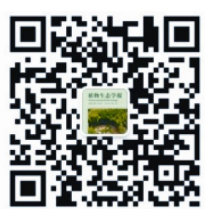

微信服务号

稿件状态查询

全文检索汶览 